\title{
Droit à la ville et contestation de l'ordre moral urbain en Turquie
}

\section{Benoit Montabone}

\section{(2) OpenEdition}

\section{Journals}

\section{Édition électronique}

URL : http://journals.openedition.org/echogeo/13567

DOI : 10.4000/echogeo. 13567

ISSN : 1963-1197

\section{Éditeur}

Pôle de recherche pour l'organisation et la diffusion de l'information géographique (CNRS UMR 8586)

\section{Référence électronique}

Benoit Montabone, «Droit à la ville et contestation de l'ordre moral urbain en Turquie », EchoGéo [En ligne], Sur le Vif, mis en ligne le 10 octobre 2013, consulté le 19 avril 2019. URL : http:// journals.openedition.org/echogeo/13567 ; DOI : 10.4000/echogeo.13567

Ce document a été généré automatiquement le 19 avril 2019.

\section{(c) (i) (9)}

EchoGéo est mis à disposition selon les termes de la licence Creative Commons Attribution - Pas d'Utilisation Commerciale - Pas de Modification 4.0 International 


\title{
Droit à la ville et contestation de l'ordre moral urbain en Turquie
}

\author{
Benoit Montabone
}

1 Les événements qui ont secoué la Turquie au mois de juin 2013 ont marqué les esprits tant par la spontanéité des rassemblements que par les mots d'ordre des mobilisations ${ }^{1}$. Durant trois semaines, le pays a connu une situation exceptionnelle, marquée par des affrontements violents dans les centres-villes entre des manifestants et la police antiémeute, mais également par une libération de la parole politique et par une occupation sous différentes formes des espaces publics. Fortement médiatisée à l'extérieur de la Turquie, passée sous silence par la plupart des médias turcs, cette mobilisation révèle autant les tensions sociales qui traversent la société turque que les modes de production de la ville en Turquie, et en premier lieu à Istanbul.

2 Il ne s'agit cependant pas d'une révolte populaire pour faire chuter le gouvernement en place et changer de régime politique, comme le qualificatif bien trop rapidement utilisé de «printemps turc » pourrait le laisser croire. Le mouvement n'a jamais élaboré un ensemble unique de revendications et ne s'est pas structuré politiquement. Durant toute la durée des manifestations, une grande partie des classes populaires et moyennes est restée fidèle au premier ministre Recep Tayip Erdoğan, au point de descendre elles aussi massivement dans la rue pour lui signifier leur soutien. Les deux journées de grève générale décrétées par plusieurs syndicats en opposition aux brutalités policières n'ont pas rencontré de grand écho au sein de la masse des travailleurs turcs. Une fois le foyer de la contestation contrôlé par la police, les manifestations ont pris d'autres formes et la vie quotidienne semble avoir repris son cours normal.

3 L'événement déclencheur du mouvement est le projet de réaménagement de la place Taksim à Istanbul par la municipalité du Grand Istanbul, projet suivi de près par le Premier ministre qui en a fait un symbole de sa politique urbaine. Un groupe d'écologistes radicaux et de citoyens opposés depuis le début au plan de réaménagement décide le 28 mai d'occuper le parc situé sur la place la veille de l'intervention des bulldozers, dans le but initial de protéger les arbres, reprenant des modalités d'action et un référentiel 
politique bien connus lors de tout conflit d'aménagement. La destruction des arbres situés sur le parc Gezi n'est alors qu'une phase opérationnelle des travaux ; les tranchées pour l'enfouissement des autoroutes urbaines étaient déjà bien avancées au début de la contestation. À la suite de l'évacuation de ce groupe par la force, des milliers de personnes se rassemblent dans la journée pour défendre le parc; ce deuxième rassemblement est lui aussi brutalement dispersé. Devant la violence de l'évacuation policière, une foule considérable se rassemble le troisième jour et décide d'occuper de manière permanente le parc Gezi. Le mouvement occupygezi se crée et obtient un succès réel sur les réseaux sociaux en Turquie et à l'étranger.

Le conflit s'étend à de nombreuses villes turques en quelques jours, et les revendications s'élargissent rapidement à la défense des modes de vie non religieux au sens large. Les centres-villes d'Istanbul, d'Ankara, d'Izmir, mais aussi d'Antalya, d'Antakya, de Bursa, de Mersin sont le théâtre d'affrontements entre manifestants et forces de l'ordre. Une recension inévitablement imparfaite des différents rassemblements montre qu'une quarantaine de villes a connu des manifestations directement reliées au mouvement occupygezi, avec des déclinaisons locales comme occupykızlay à Ankara ${ }^{2}$. Le discours fortement répressif du Premier ministre alimente la contestation, qui s'autonomise et se dote d'une identité propre par une formidable créativité artistique ${ }^{3}$. En parallèle, les affrontements font 4 morts officiels, 3 parmi les manifestants et 1 parmi la police. Après deux semaines d'affrontements et de crispations, le gouvernement fait évacuer la place Taksim le 11 juin et cherche à étouffer le mouvement. Ce dernier se prolonge alors sous d'autres formes, dont la tenue d'assemblées citoyennes dans plusieurs parcs d'Istanbul, d'Ankara et d'Izmir, et dans une moindre mesure dans une vingtaine de villes du pays. Le 3 juillet, un tribunal d'Istanbul invalide le projet d'aménagement de la place Taksim invoquant une incompatibilité juridique avec les plans d'urbanisme réglementaires. Ainsi, un mouvement populaire en partie spontané a réussi à faire échouer un projet d'aménagement du gouvernement, soulignant les limites de son mode de transformation des villes.

En 2009, nous soulignions déjà dans cette rubrique le caractère artificiel et forcé des stratégies d'internationalisation de la ville, arguant du décalage grandissant entre la politique de grands projets fantasmagoriques promus par le parti au pouvoir avec les attentes d'une partie de la population (Candelier-Cabon, Montabone, 2009). Depuis une dizaine d'années, la production scientifique s'est également faite écho du processus brutal de néolibéralisation de la ville et de standardisation des espaces publics, transformés petit à petit en espaces de consommation et de mise en scène d'une culture facilement internationalisable aussi bien en Turquie (Karaman, 2008; Pérouse, 2007; Erkip, 2003) qu'ailleurs dans le monde (Brenner, Theodore, 2002; Harvey, 2008; Zukin, 2010; Morange, 2011;). En Turquie, cette politique de transformation urbaine est marquée par la volonté d'éradiquer les quartiers hérités du processus informel d'urbanisation, incarné par la forme morphologique et sociale du gecekondu (Pérouse, 2004 ; Cankat, 2010). Elle s'est accélérée ces dernières années, et s'est diffusée depuis les grands centres urbains servant de vitrine à la politique de développement de l'AKP vers l'ensemble des strates de la hiérarchie urbaine du pays. Cette politique de renouvellement urbain (kentsel dönüşüm) s'effectue selon des modalités maintenant connues et qui se répètent dans de nombreuses opérations (Uzun, Çete, Palancioğlu, 2010), ce qui permet d'affirmer qu'il s'agit bien d'une volonté politique de mener un large programme cohérent de remodelage des villes - de leur forme architecturale comme de leur composition sociale. 
6 L'argument principal développé ici est que ces transformations urbaines sont indissociables du projet social du gouvernement turc, et que cette relation est au cœur de la contestation du mois de juin 2013. Cet article ne prétend pas apporter une lecture exhaustive de cette dernière. Il vise plutôt à en donner une interprétation géographique, en s'appuyant sur de nombreuses sources de presse écrite, sur des décryptages de pages personnelles sur les réseaux sociaux et sur des entretiens informels avec des acteurs directement impliqués dans le mouvement.

\section{Retour sur une mobilisation exceptionnelle}

7 La chronologie des événements a été rapidement abordée en introduction. Un compterendu précis, détaillé et circonstancié est bien sûr utile, mais il a déjà été fait par ailleurs et ne constitue pas le cœur de cette réflexion ${ }^{4}$. Pendant le mouvement, M. Dikeç a publié un texte remarqué montrant le lien entre le positionnement politique de l'AKP depuis dix ans et l'éclatement spontané de la colère urbaine (Dikeç, 2013). Peu après celui-ci, J.F. Pérouse démontrait que la colère du parc Gezi révélait une transformation fortement politique (Pérouse, 2013). Sans occulter cette dimension politique incontournable, il est pour nous nécessaire de revenir sur des éléments clé de cette mobilisation pour en comprendre la dimension intrinsèquement urbaine.

\section{«Ce n'est pas une question d'arbres... »}

8 Si l'élément déclencheur est un projet d'urbanisme sur une place bien particulière à Istanbul, le mouvement ne peut être résumé à un simple conflit d'aménagement. Le parc Gezi en lui-même n'était pas un lieu particulièrement investi par la population avant son projet de destruction, ni par les riverains ni par les passants. Lieu de deal et de prostitution nocturnes, il était plutôt malfamé et évité par une grande partie de la population venue par la suite le défendre. Le réductionnisme écologiste fait notamment par le gouvernement turc mais également par des sympathisants du mouvement masque mal l'ampleur de la contestation, dont un des slogans était justement «Ce n'est pas une question d'arbres; c'est une question de démocratie ». Il s'agit bien d'un mouvement de contestation de fond contre les dérives autoritaires, conservatrices et islamistes du gouvernement turc, dont la somme accumulée ces cinq dernières années devient difficile à énumérer. Au cours du printemps 2013, la polémique sur la consommation d'alcool, la limitation drastique de la vente de la pilule du lendemain, le discours moralisateur sur le modèle des femmes comme mère de trois enfants auront bien plus pesé dans la mobilisation que la centaine d'arbres du parc Gezi.

Cependant, la part du conflit d'aménagement ne doit pas être minorée. La rapide mobilisation sur cette place s'explique aussi par l'existence de réseaux bien organisés et habitués aux protestations contre des projets urbains jugés contraires à l'intérêt général de la population et de la ville comme cadre de vie. Dès l'annonce par le gouvernement d'un projet de réaménagement de la place, une plateforme citoyenne s'est constituée pour contester la dimension idéologique du projet et proposer des scénarios alternatifs, en organisant des symposiums et menant des campagnes de presse assez efficaces dans les milieux libéraux ${ }^{5}$. On retrouve dans cette plateforme un ensemble d'architectes et d'urbanistes très investis sur la question de la légitimité urbaine, notamment la Chambre des Architectes et des Planificateurs Urbains d'Istanbul, qui mènent depuis dix ans des 
actions contre la néolibéralisation de la ville et pour une politisation des mobilisations locales (Petit, 2011). En 2005, cette Chambre professionnelle avait fait annuler le projet Dubaï Towers porté par l'émir de Dubaï Rachid El Makthoum et facilité par le Premier ministre turc et le maire d'Istanbul. Ce jugement avait porté un coup d'arrêt aux investissements des pays du Golfe dans l'immobilier à Istanbul. C'est également un membre de cette Chambre qui est devenu porte-parole éphémère du mouvement occupygezi pendant quelques semaines, utilisant ses compétences professionnelles et ses réseaux, notamment judiciaires, pour contester le projet devant le tribunal d'Istanbul.

\section{Une mobilisation avant tout urbaine}

10 La mobilisation de juin 2013 est intrinsèquement urbaine. Elle s'est bien sûr cristallisée sur un conflit urbain et s'est exprimée massivement en ville, ce qui n'est pas étonnant étant donné le taux d'urbanisation du pays. En outre, elle s'est surtout effectuée dans des lieux concentrant et représentant le mieux l'urbanité occidentale, dans les centres-villes commerçants et sur les places symboliques de chaque ville : Taksim à Istanbul, mais aussi Kızılay à Ankara et Alsancak à Izmir. L'action revendicatrice en elle-même est en effet circonscrite à de petits espaces, occupant une infime portion des espaces urbains tout en restant spectaculaire. Le campement sur la place Taksim est le seul à avoir été installé de manière pérenne, produisant une microgéographie des mouvements de gauche, nationalistes, altermondialistes et revendicatifs de Turquie. Les participants ont tous souligné l'extraordinaire ambiance de ce lieu de vie devenu espace de publicisation des revendications, mêlant parfois avec quelques accrocs associations LGBT, mouvements d'extrême gauche nationalistes et partis politiques kurdes. La forte intensité politique et sociale de ces lieux éphémères ne doit pas pour autant masquer la réalité : l'espace urbain dans son ensemble n'a pas été contrôlé par les manifestants, et même au plus fort des affrontements la vie urbaine quotidienne a suivi son cours. Les touristes ont continué de déambuler dans les allées du grand bazar, ajoutant pour les plus téméraires d'entre eux une visite sur la place Taksim investie et transformée en agora.

11 Les manifestations de rue, occupations de places et édifications de barricade ne sont cependant pas la seule forme d'expression du mouvement. Un grand nombre de personnes mobilisées n'est pas venu sur la place Taksim et n'a pas participé aux grandes manifestations, car elles en étaient empêchées pour différentes raisons : lieu de résidence très éloigné du théâtre des affrontements, obligations professionnelles ou familiales, crainte de la foule et surtout de la police anti-émeute. La violence de la répression, l'utilisation massive de gaz (qui a donné lieu à un nombre incroyable de jeux de mots, de slogans et de créations artistiques) a dissuadé de nombreux Turcs de descendre dans la rue.

12 Mais au-delà des témoignages de sympathie sur les réseaux virtuels, des formes de mobilisation originales se sont multipliées à travers de nombreux quartiers, y compris dans des quartiers réputés conservateurs et fidèles au gouvernement. Des regroupements spontanés se sont créés à la sortie du travail ou du lycée pour les plus jeunes pour discuter de la situation; on a vu des voisins descendre sur le pas de la porte et boire un verre (de boisson alcoolisée) dans la rue en signe de défiance vis-à-vis du gouvernement. Tous les soirs, de gigantesques concerts de casserole ont envahi l'espace sonore, se joignant aux effets de lumière des résidents qui allumaient et éteignaient pendant des dizaines de minutes leurs éclairages domestiques, faisant clignoter la ville. Le paysage 
urbain, tant visuel que sonore, est ainsi entré en résistance, permettant de déceler à la nuit tombée les quartiers les plus vindicatifs. On peut $\mathrm{y}$ ajouter les sirènes de certains bateaux sur le Bosphore, les drapeaux turcs aux fenêtres et surtout l'image omniprésente d'Atatürk, brandi comme le symbole retrouvé de la laïcité nationale. Certes les drapeaux et les portraits d'Atatürk ont toujours fait partie du paysage urbain en Turquie ; mais l'affichage du fondateur de la République comme figure tutélaire a permis d'affirmer l'attachement de toute une frange de la société aux fondements républicains et laïcs du pays.

\section{Sens des lieux et maîtrise des espaces symboliques}

13 La place Taksim d'où l'ensemble du mouvement est parti et qui le symbolise encore aujourd'hui est un espace enjeu, qui incarne la continuité des luttes urbaines à Istanbul et symbolise l'emboitement des temporalités propres à la mobilisation (Bonny et al., 2011). Elle est un haut lieu pour Istanbul et au-delà pour toute la Turquie, dont la dimension symbolique est essentielle pour expliquer la propagation $\mathrm{du}$ mouvement $\mathrm{du}$ printemps 2013. Il s'agit tout d'abord d'un lieu emblématique de la République de Turquie, sur lequel se trouve le monument dédié aux héros de la guerre d'indépendance d'un côté, et le centre culturel Atatürk de l'autre, symbole de l'ouverture culturelle occidentale du pays pendant la deuxième moitié $\mathrm{du} \mathrm{XX}^{\mathrm{e}}$ siècle. Taksim est aussi un marqueur de la modernisation urbanistique du pays et de la ville. Même si elle n'occupe pas une place centrale dans le plan Prost de $1936^{6}$, elle est le point de redistribution des flux vers le nord de la ville et la porte d'entrée du quartier des affaires. Il s'agit d'un espace symbole sur lequel l'ensemble des gouvernements successifs ont tenté d'imposer leur marque, et qui selon l'urbaniste Koran Gümüş est «le site de lutte privilégié pour la domination idéologique dans la ville $»^{7}$.

Taksim est en effet un espace emblématique des luttes politiques et syndicales en Turquie. À la suite des manifestations violentes du $1^{\text {er }}$ mai 1977 qui ont fait 6 morts, la place $\mathrm{a}$ été interdite de toute manifestation jusqu'au $1^{\mathrm{er}}$ mai 2010 , où pour la première fois le défilé a été autorisé par les autorités. Entre ces deux dates, tous les ans, les organisations syndicales et les partis politiques de gauche et d'extrême gauche ont organisé une marche sur Taksim à partir des trois grandes artères qui y mènent, cortèges inlassablement bloqués par la police et entraînant des heurts pendant plusieurs heures. Ce rituel est devenu un véritable processus de socialisation militante autant qu'une guerre idéologique pour reconquérir l'espace (Uysal, 2008). Cet héritage a une part importante dans l'explication des manifestations du printemps, car cette année, du fait des travaux en cours, les manifestations du $1^{\mathrm{er}}$ mai ont été interdites sur Taksim. Occuper Taksim, chercher à y revenir coûte que coûte tous les soirs, rappelle ce long processus militant et montre la volonté de conserver un acquis dans la lutte pour la maîtrise des espaces symboliques à Istanbul (illustration 1). 
Illustration 1- Fresque murale représentant la marche sur Taksim lors du $1^{\mathrm{er}}$ mai 2010

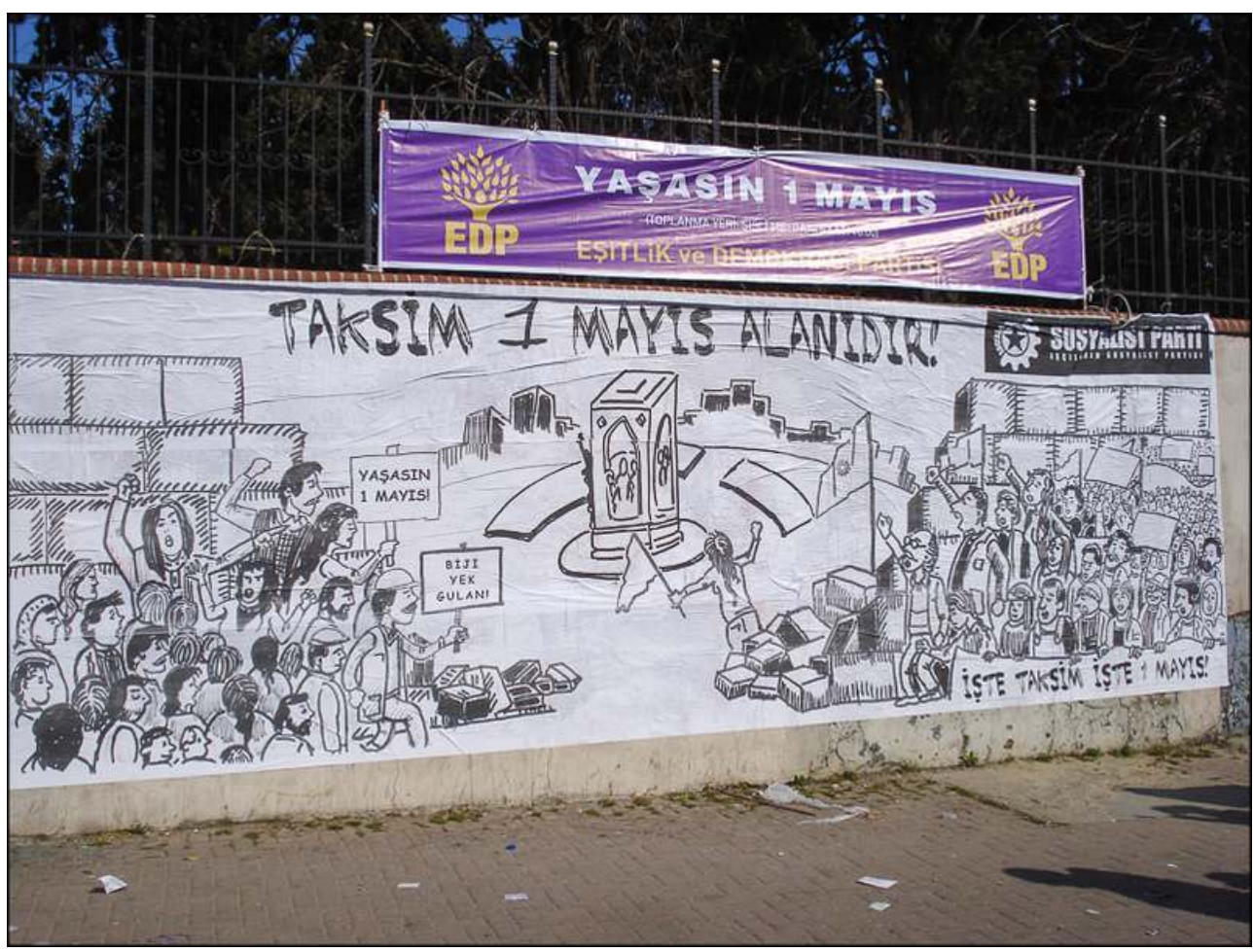

Auteur : B. Montabone, 2010.

\section{Une explication par l'écologie urbaine}

La manière dont les mobilisations brutales se créent dans un espace donné, et surtout la manière dont elles sont façonnées par l'espace dans lequel elles émergent et se déploient est une question centrale dans l'analyse des mouvements protestataires urbains. Comme le souligne Javier Auyero, «L'espace et le lieu déterminent, tout en les favorisant, l'émergence des mouvements de contestation; en retour, ceux-ci contribuent à actualiser les potentiels qu'offre l'espace tout en le restructurant» (Auyero, 2005, p. 126). Les travaux emblématiques de cette approche, comme ceux de R. Gould sur la Commune de Paris (Gould, 1995) ou ceux de D. Zhao sur le mouvement étudiant chinois de 1989 (Zhao, 1998), montrent qu'une forme d'organisation matérielle de l'espace urbain favorise la mobilisation protestataire (Lefebvre, 1970; Mathieu, 2011). Le dispositif spatial, qui est toujours situé au sein d'un tissu de relations sociales (Auyero, 2005), favorise les stratégies d'occupation du terrain, notamment par l'érection de barricades, et permet une forte concentration de population tout en faisant apparaître des espaces de sociabilité. Il ménage également des espaces de refuge en cas d'attaque de la police. L'importance de ce dispositif spatial peut se voir dans les stratégies anti-émeutes de cette dernière, qui vise non pas à occuper l'ensemble de l'espace mais à briser les relations et les échanges qui le constituent. Barrages routiers, fermeture de certains accès (physiques et virtuels), arrêt des transports en commun, arrestations ciblées sur les porteurs d'information sont autant d'éléments qui visent à briser les connexions entre les individus et les espaces porteurs de la mobilisation (Mathieu, 2011). Les événements de 
juin se prêtent assez bien à ce cadre d'analyse, notamment le quartier de Beyoğlu au centre d'Istanbul, objet d'un véritable siège pendant quelques jours (illustration 2).

Illustration 2 - Taksim : le dispositif spatial de la mobilisation

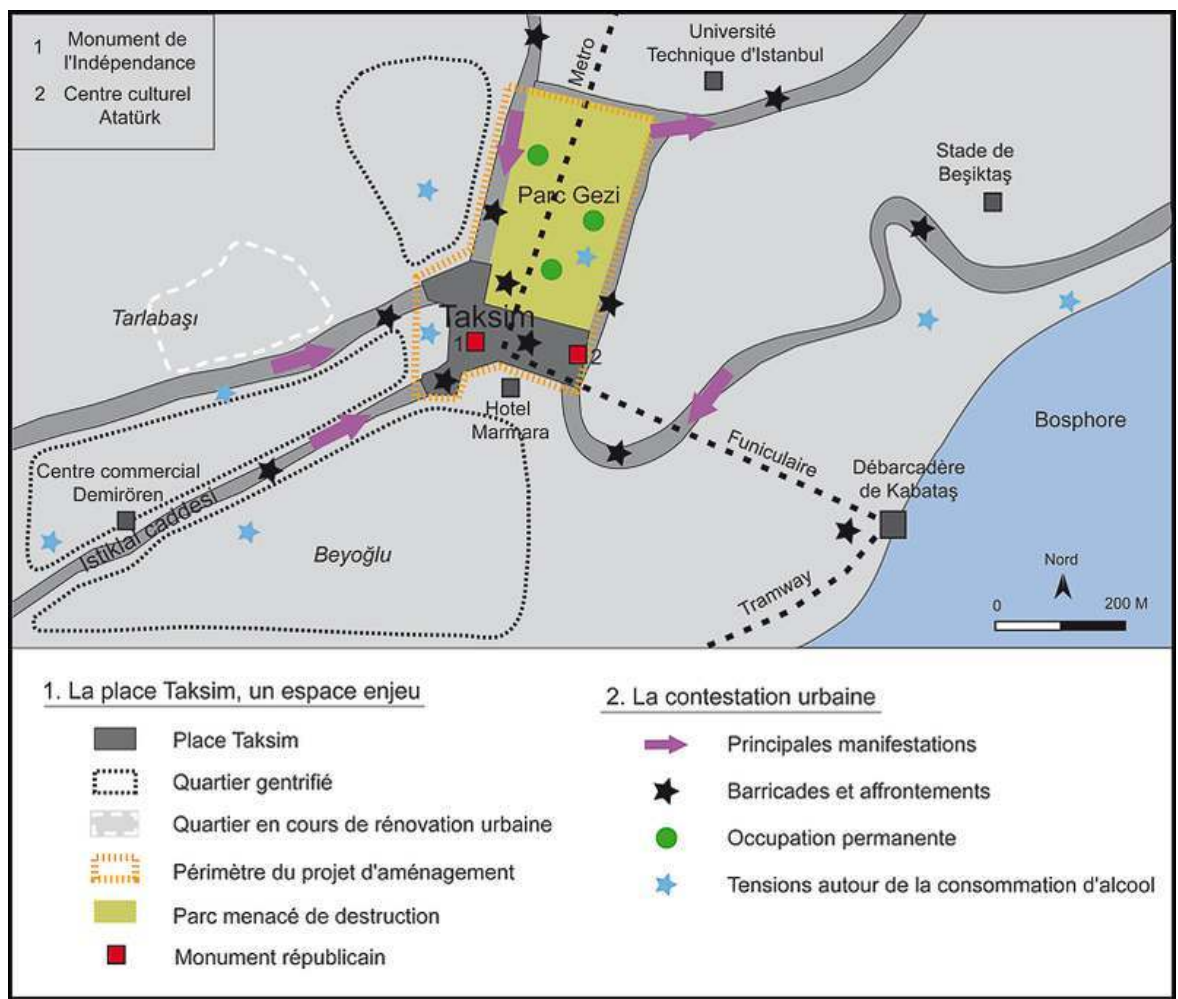

Auteur : B. Montabone, 2013.

Le quartier qui entoure la place Taksim est en effet au cœur du dispositif spatial de la mobilisation. Le témoignage suivant, donné par une résidente francophone du quartier, témoigne bien de cette écologie urbaine de la mobilisation :

«J'étais hier soir au parc et sur l'avenue principale jusqu'à 20h00/20h15, l'atmosphère était bon enfant, ressemblant plus à une kermesse qu'à autre chose, le parc et les environs étaient bondés de monde, familles avec enfants, landaus, jeunes et vieux, touristes etc.... Ça a complètement basculé un peu avant $21 \mathrm{~h} 00$ avec l'arrivée de la police dans le parc et sur la place avec forces de l'ordre et bulldozers. Maintenant à l'heure où je vous écris (du quartier de Tophane), les violences continuent, combats d'Osmanbey à Taksim (centreville côté européen) entre manifestants et police, manifestations dans le centre-ville et dans le quartier de Besiktas, les gens depuis l'aube, par flux, se dirigent vers le centreville de Taksim, totalement bouclé et vers Besiktas. (...) Les rues sont toujours pleines d'effluves de gaz lacrymogènes et il y en a tant que nous sommes obligés de fermer les fenêtres des différents lieux où je passe ${ }^{8}{ }^{8}$

On le voit, la proximité est un facteur central dans la mobilisation et dans la diffusion de la contestation. Une proximité spatiale bien sûr: les barricades, émeutes et manifestations ont eu lieu essentiellement à Beyoğlu, sur l'avenue Istiklal, à Beşiktaş et Nişantaşı, les quartiers attenant immédiatement à la place Taksim. Cette proximité spatiale est aussi sociale, ces quartiers étant le lieu privilégié de résidence des classes moyennes intellectuelles, des étudiants et actifs diplômés qui ont été les fers de lance de 
la contestation. À ces deux proximités classiques s'ajoute une proximité culturelle : il s'agit d'un espace à l'identité urbaine bien marquée, fortement gentrifié, où sont implantés de nombreux commerces de la culture alternative et underground de la ville (librairies, cafés, studios, salles de concert, etc.). Les réseaux militants et de manière plus large la sociabilité alternative se sont tissés dans ces quartiers, ce qui explique la mobilisation exceptionnelle et rapide des manifestants. Ces quartiers sont également des lieux de rassemblement traditionnels des supporters des équipes de football d'Istanbul, eux aussi habitués aux combats de rue avec la police. Les manifestants ont donc une grande maîtrise de l'espace dans lequel ont eu lieu les affrontements majeurs, leur servant à la fois d'espace théâtre et d'espace refuge.

19 La configuration de l'espace urbain et surtout la maitrise d'un espace assez homogène dans ses pratiques spatiales et sociales ont donc été déterminantes. Il s'agit ici plus de pratique que de résidence, car ces quartiers sont plus hétérogènes qu'on ne pourrait le croire de premier abord. Ceux qui pratiquent cette culture occidentale et alternative habitent souvent ailleurs, parfois loin, mais maîtrisent parfaitement l'espace en question et ses codes sociaux, quand certains résidents en sont exclus ou en restent volontairement à l'écart.

Cependant, l'analyse du mouvement par l'écologie urbaine reste insuffisante, notamment du fait des différentes formes de mobilisation dont nous avons parlées précédemment et de la diffusion du mouvement au-delà des trois espaces centraux d'Istanbul, d'Ankara et d'Izmir.

\section{La contestation de l'ordre moral urbain}

21 Au-delà de leur propre temporalité, ces manifestations ne sont en effet pas arrivées par hasard; elles répondent à une conjonction d'éléments politiques et sociaux qui se cristallisent dans l'affrontement, mais qui continuent d'exister en dehors du théâtre des manifestations. Ces dernières ont créé des espaces-temps de tension bien particuliers, dans lesquels s'est incarné le refus par toute une frange de la population turque de l'islamisation rampante des institutions et de la société.

Ainsi, si la mobilisation a essaimé au-delà du parc Gezi, c'est essentiellement parce que ce projet d'aménagement cristallise l'ensemble de l'évolution de la politique urbaine de l'AKP depuis son arrivée au pouvoir en 2002. Cette politique avait déjà commencé quand le Refah, parti islamiste dissout en 1998 par la Cour Constitutionnelle dont est issu l'AKP actuel, avait remporté les élections municipales de 1994 à Istanbul. Le ré-aménagement de la place Taksim concentre en effet les trois piliers de la politique de l'AKP ces dix dernières années: la création d'un immense centre commercial comme symbole du néolibéralisme économique, un projet de mosquée (finalement abandonné) comme symbole du conservatisme religieux, et la reconstruction d'une caserne ottomane comme symbole du néo-ottomanisme culturel. À travers la contestation de ce projet, c'est un ensemble de mesures sociales, sociétales, économiques et politiques qui ont été remises en cause. 


\section{Néolibéralisme économique} ouvertement l'économie libérale de marché et fait appel à la réussite individuelle comme preuve du développement sans précédent du pays. L'AKP s'appuie sur une assise sociale assez large, composée essentiellement de petits commerçants et d'entrepreneurs anatoliens, très sensibles au discours politique fondé sur des références constantes au religieux. Les réseaux politiques sont étroitement imbriqués avec les réseaux d'affaires, et le gouvernement est ouvertement soutenu par de grands groupes turcs plutôt récents, constitués depuis une trentaine d'années par des entrepreneurs provinciaux (Dilek, 2012). Cette politique néo-libérale passe par un retrait de l'État d'un certain nombre de domaines clé, ou tout du moins par leur ouverture à la concurrence du secteur privé : santé, enseignement supérieur. Cela passe également par des privatisations massives (industries, aéroports, etc.), notamment la privatisation emblématique des manufactures d'alcool et de tabac (TEKEL) en 2008, qui s'était soldée par la fermeture de 12 unités de production dans le pays.

Certes cette politique néolibérale n'est pas l'apanage de l'AKP; le tournant est souvent situé par les économistes turcs à l'ère Özal, Premier ministre de l'ouverture économique du pays entre 1983 et 1989 (Yalman, 2009). Mais l'AKP, fortement encouragée par le FMI et la Banque mondiale à la suite de la crise monétaire de 2001, a poussé le modèle très loin et se pose en championne du développement économique, synonyme dans son discours de mieux-être pour l'ensemble du pays donc de la population. Cette notion de développement est centrale pour comprendre le projet politique de ce parti, qui la porte dans son nom; il s'agit d'un développementalisme d'État qui se mesure en kilomètres d'infrastructures construits', formant un néolibéralisme régulé, et qui satisfait une grande partie de la population turque "fière devoir arriver la modernité au village", constituant en même temps une forme de néolibéralisme social (Öniş, 2012).

Ce néolibéralisme économique se voit de manière criante dans la production de la ville, que les chercheurs en sciences sociales turcs et étrangers déconstruisent depuis plus d'une quinzaine d'années. Sur la production immobilière elle-même tout d'abord, on observe une accumulation de grands projets somptuaires déconnectés du marché local et qui se vend à l'international, comme le fameux projet Bosphorus City qui a obtenu le prix du meilleur projet turc au MIPIM ${ }^{10}$ de Cannes cette année (illustration 3). Ces projets aux noms évocateurs (FinansKent, la ville de la finance par exemple) dessinent une nouvelle géographie urbaine, faite de polarités périphériques reliées entre elles par des autoroutes urbaines et branchées sur les circuits internationaux grâce à leur accès facile aux aéroports. Cette politique de transformation urbaine se voit aussi clairement dans la récupération de nombreux espaces centraux occupés illégalement par des gecekondu, dont le procédé de renouvellement assure de fortes plus-values foncières aux promoteurs immobiliers (Kuyucu, Ünsal, 2010). 
Illustration 3 - « Bosphorus City » dans l'arrondissement de Küçükçekmece à Istanbul, projet immobilier primé au MIPIM de Cannes en 2013

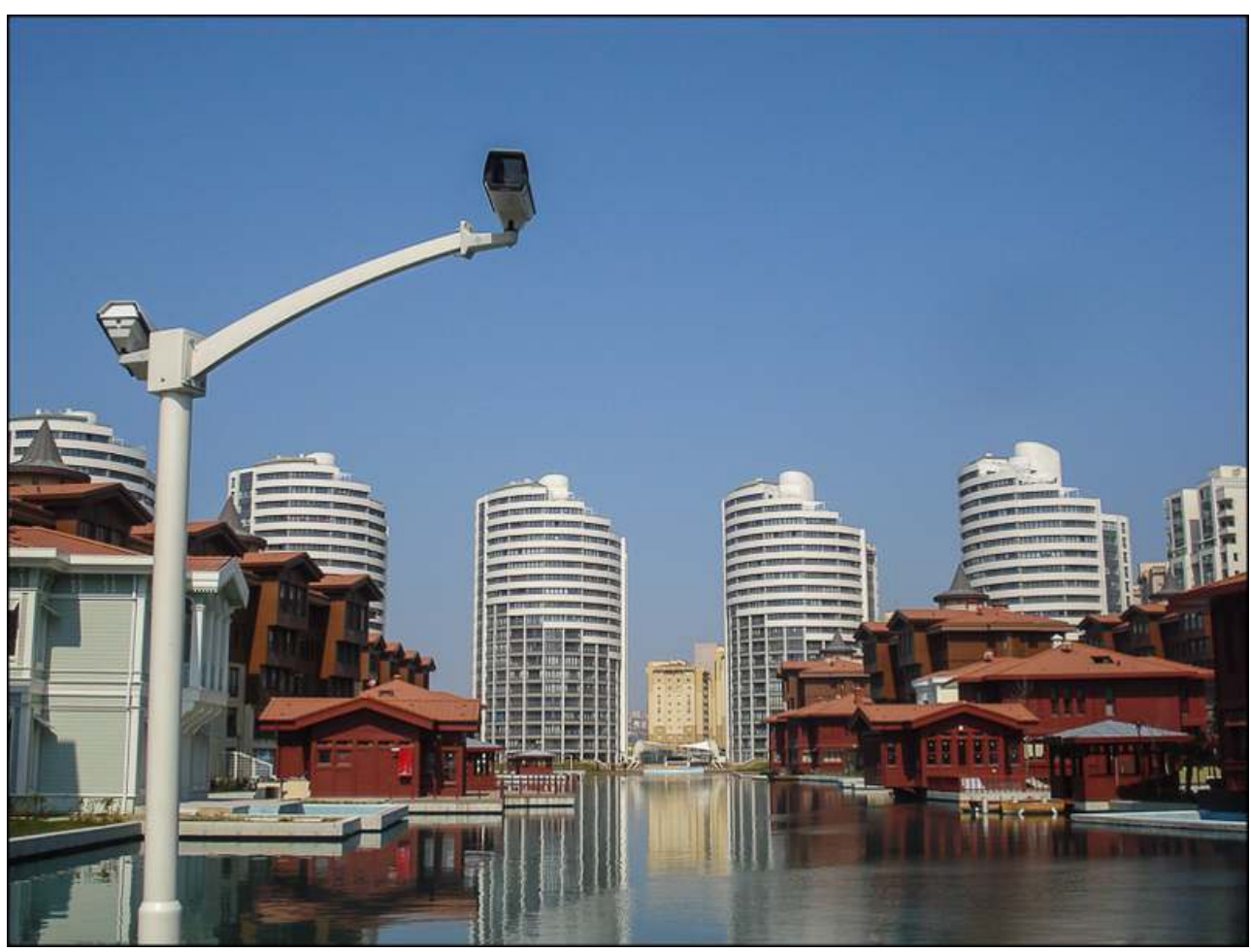

Auteur : B. Montabone, 2012

Une place particulière doit être faite ici à la course aux malls et autres centres commerciaux plus ou moins spécialisés tout au long des autoroutes urbaines, dont certains sont consacrés à la voiture. Ici aussi, dresser une liste exhaustive des centres commerciaux ouverts dans les cinq dernières années en Turquie serait impossible. Symboliquement, le centre commercial Demirören sur l'avenue Istiklal d'Istanbul concentre l'ensemble des griefs pouvant être fait à cette frénésie du shopping mall: exacerbation du consumérisme dans ses formes, mais aussi non-respect des règles d'urbanisme et entente entre milieux d'affaire et milieux politiques pour sa construction ${ }^{11}$. Ce néolibéralisme économique appliqué à la production de la ville entraîne ainsi la construction d'un nouveau paysage urbain, et en son sein de nouveaux espaces publics basés essentiellement sur la consommation (Erkip, 2003 ; 2005).

\section{Conservatisme religieux}

L'AKP est un parti islamiste. Présenté par de nombreux observateurs comme la branche modérée de l'islam politique, il n'en reste pas moins que les références religieuses sont omniprésentes dans ses discours et dans ses actes, de manière plus ou moins évidente. Cette référence religieuse est visible dans l'espace public et dans la société dans son ensemble. Sur le plan social, il s'agit tout d'abord d'un discours récurrent sur les valeurs traditionnelles de la religion, et par ricochet de la société turque dans son ensemble. Dieu et le Coran sont cités en exemple, et le Premier ministre prend un malin plaisir à utiliser systématiquement des mots ou expressions d'origine arabe fortement connotés, quand leur synonyme turc existe bel et bien dans la langue officielle. Jean-François Pérouse a 
très bien décrit comment le Refah, lors de son arrivée au pouvoir municipal à Istanbul en 1994, utilisait systématiquement certains mots pour marquer sa filiation religieuse : ainsi le mot hakk pour droit et nom hukuk, le hakk étant le droit naturel, historique et divin quand le hukuk est le droit juridique et politique (Pérouse, 1999).

Cette année, le débat politique s'est cristallisé sur la question de la consommation de l'alcool, le gouvernement ayant fortement restreint les horaires et lieux de vente de boissons alcoolisées en le justifiant par un discours de santé publique, quand ce n'était pas moralisateur. Déjà la disparition des boissons alcoolisées sur les vols internes de la compagnie nationale Turk Hava Yolları (Turkish Airlines) en 2012 avait fait bondir la Turquie laïque; le discours d'avril 2013 sur la bière et l'ayran a fortement ravivé les tensions ${ }^{12}$. Le discours sur la place des femmes dans la société est également controversé. De nombreuses femmes voilées et non voilées ont participé aux manifestations du mois de juin, soucieuses de la condition des femmes dans ce raidissement sociétal, symbolisé par la question du port de foulard. Un cadre de l'AKP avait par exemple déclaré sur facebook en 2011 « qu'une femme sans voile était comme une maison sans rideaux : soit elle est à vendre, soit elle est à louer $\aleph^{13}$, suscitant un vaste débat sur la considération de la place des femmes au sein du parti au pouvoir. À cela s'ajoute les incitations pour être une bonne épouse et une bonne mère, devant faire au moins trois enfants pour remplir son rôle envers sa famille et son pays. Ce conservatisme religieux sexiste trouve son pendant dans l'aménagement de la ville.

L'interprétation de l'islam par la mouvance AKP se retrouve en effet dans la fabrication des espaces publics. L'image de la pureté est souvent associée à celle de la salubrité, et audelà de la propreté des rues et des jardins. De même, l'environnement est souvent ramené à son seul cadre physique et hygiéniste : des espaces verts et fleuris bien agencés, opposés aux espaces de friche, aux rues sinueuses et sales, aux espaces vacants en général. Sur le plan de la politique familiale, la ville anarchique, auto-construite ne laisse pas de place aux familles : les enfants ne peuvent pas jouer dans la rue, les personnes âgées ont une mobilité fortement altérée, etc. Il faut donc construire des espaces, des parcs très stéréotypés pour la plupart, où les enfants peuvent jouer en sécurité, où les personnes âgées peuvent se déplacer plus facilement, où les familles peuvent se retrouver autour d'un barbecue, c'est-à-dire des lieux où ces dernières ne sont pas menacées par les travers de l'urbanité. En parallèle, ces parcs sont censés rassurer les mères dans leur rôle de mère, et en même ils assignent les femmes à un rôle de mère. Bien évidemment, la consommation d'alcool dans ces espaces publics est très mal vue, la plupart du temps interdite et réprimée par les polices municipales. L'aménagement des espaces publics est ainsi au cœur du projet social de l'AKP, qui gère une grande majorité des villes de Turquie (illustration 4). 


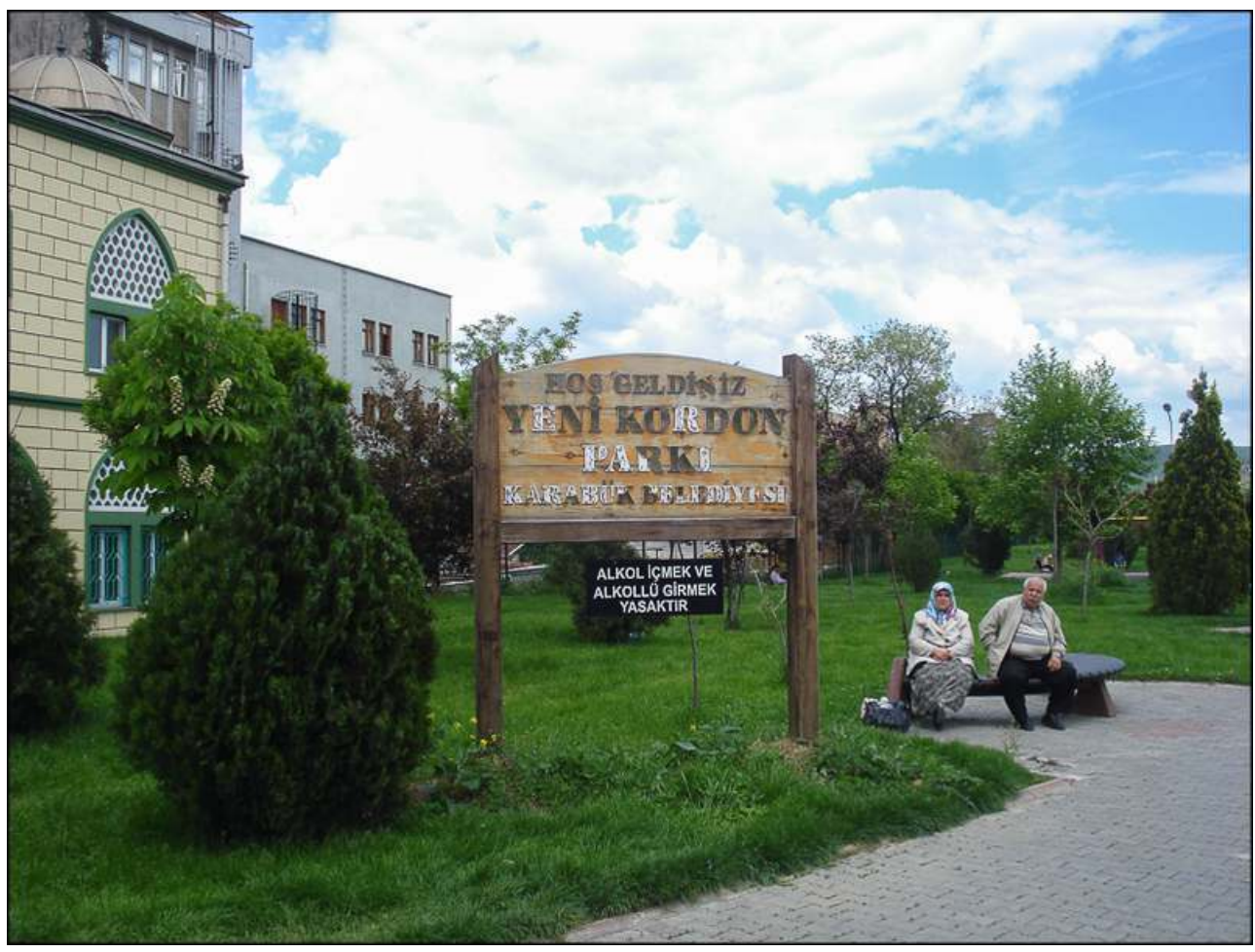

Sur la pancarte, on peut lire : « Il est interdit de boire de l'alcool et de pénétrer alcoolisé dans le parc ». Auteur: B. Montabone, 2011.

\section{Néo-ottomanisme culturel}

Ce qu'on appelle néo-ottomanisme dans la politique de l'AKP est un ensemble de mesures, de déclarations et de prises de position dans la politique extérieure de la Turquie ces cinq dernières années (Artik, 2013; Öktem et al., 2012) Elles ont pour but de permettre à la Turquie de retrouver une influence diplomatique et un poids géopolitique dignes de l'Empire ottoman, avec d'un côté une politique volontariste vers l'Europe (implication dans les Balkans et agenda européen) et de l'autre l'investissement du Moyen-Orient au sens large par les acteurs politiques, économiques et religieux de Turquie (soutien aux Frères Musulmans en Égypte, investissements massifs en Irak du Nord et en Syrie avant la guerre civile, présentation du pays comme un modèle politique pour les islamistes modéré du Maroc et de Tunisie, positionnement dans la reconstruction de la Libye, etc.).

31 Sur le plan intérieur, ce néo-ottomanisme se traduit par une mise en scène culturelle et un détournement du patrimoine historique à des fins idéologiques. La rénovation des quartiers anciens de la péninsule historique et de Beyoğlu, comme Sülemaniye ou Tarlabaşı, à proximité immédiate de Takism, s'effectue par un façadisme affirmé qui met en valeur un style architectural ottoman réinventé, au détriment des différentes époques successives qui forment le tissu urbain du vieil Istanbul (illustration 5). 


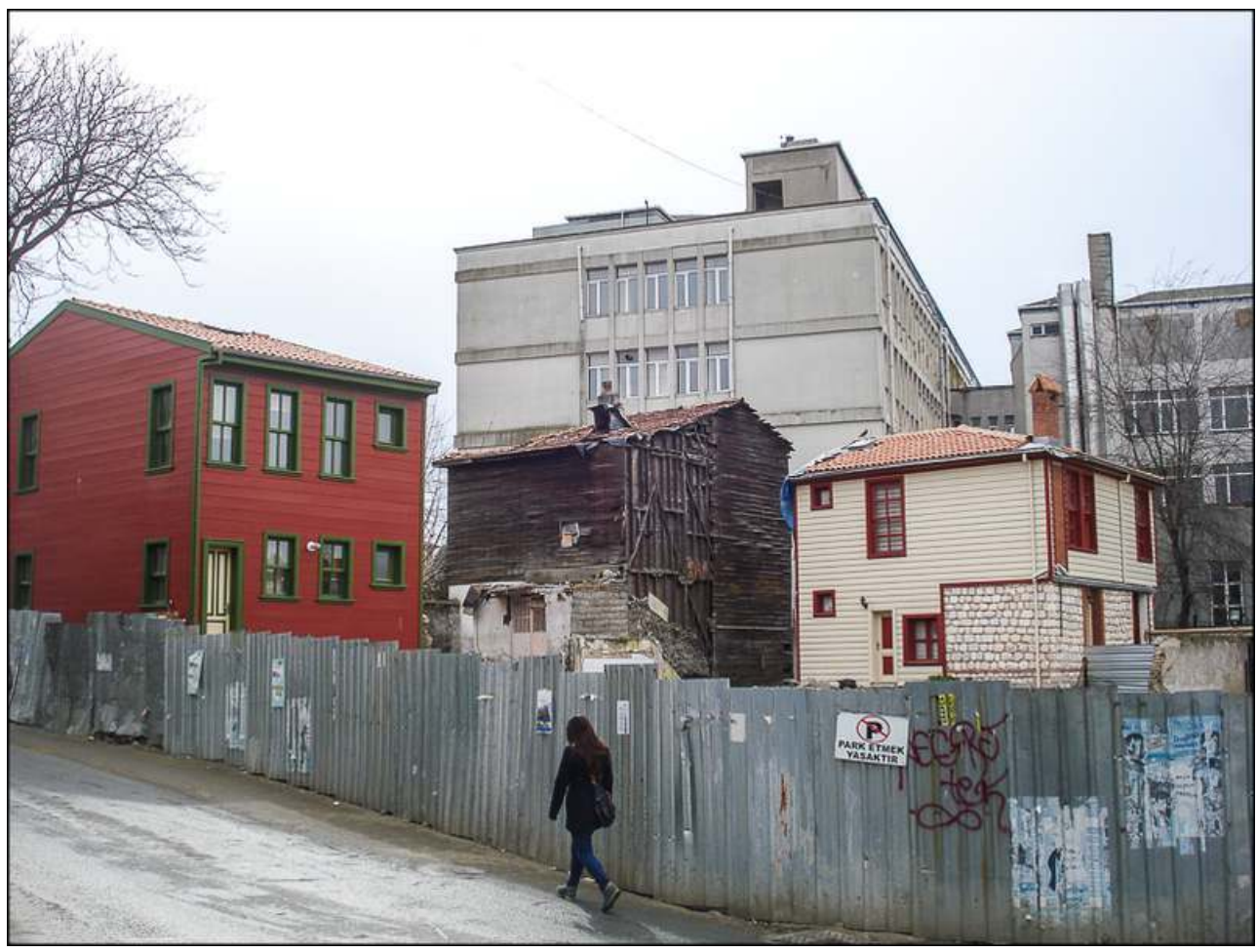

Auteur : B. Montabone, 2013.

À défaut d'être restaurée et entretenue, la muraille de Théodose II est instrumentalisée pour glorifier l'histoire ottomane de la ville. Sa conquête par Mehmet II est commémorée tous les ans à grand renfort de musique militaire traditionnelle, et un musée présentant le siège de Constantinople a été édifié de façon permanente à ses pieds. Cette néoottomanisation se voit également par la «mise en tulipe » de la ville, en référence à la période faste de l'Empire ottoman dite des tulipes ${ }^{14}$. Des millions de bulbes sont plantés chaque année (le record est d'ailleurs dépassé tous les ans à la grande fierté du maire), et de manière révélatrice le Premier ministre s'est empressé de faire planter des tulipes dans le parc Gezi fraîchement évacué. Un élément récent de cette néo-ottomanisation culturelle a contribué à alimenter le climat de défiance envers le gouvernement au printemps 2013 : la dénomination du troisième pont sur le Bosphore du nom de Sultan Selim $1^{\text {er }}$ (dit le terrible), conquérant glorieux de l'Empire ottoman entre 1512 et 1520 , mais également pourfendeur des hérésies chiites et massacreur des alévis.

Cette évolution de la politique culturelle se concentre dans le projet de réaménagement de la place Taksim : le but est de piétonniser la place, de la rendre plus vivable pour les familles et abordable pour les consommateurs, d'y construire une grande mosquée et un grand centre commercial ultra-moderne. Ce dernier devait occuper une réplique contemporaine de la caserne ottomane de Halil Pasha, symbole du coup d'État avorté d'une partie de l'élite ottomane pour empêcher l'évolution vers un Empire constitutionnel en 1909. Il s'agissait donc de prendre une revanche néo-ottomane sur la République laïque et de lisser un peu plus l'espace public au profit d'un consumérisme chevronné, le tout encadré par de nouveaux minarets (qui bien sûr devaient initialement dépasser le clocher de l'église chrétienne voisine). 

ottomanisme culturel) constituent ainsi l'ordre moral urbain de l'AKP, où les projets économique, social et culturel s'identifient dans l'espace public. On retrouve ces éléments dans tous les aménagements des espaces urbains en Turquie, du simple parc au nouveau quartier périphérique de type grand ensemble, avec des indices plus ou moins flagrants, et dont les minarets sont la plupart du temps des marqueurs spatio-temporels forts (Batuman, 2013). La dimension hygiéniste est claire, et couplée à une surveillance panoptique des comportements. Il s'agit de modeler la communauté de vie (assimilée à la communauté de croyants) par l'espace, de faire en sorte que l'espace physique accompagne le modelage de la communauté selon un idéal religieux, et ainsi que l'espace public offre à la société l'image à laquelle elle doit se conformer.

\section{Conclusion : droit à la ville et reconquête des espaces publics}

La contestation en Turquie s'est élevée contre cet ordre moral urbain. Au-delà du parc Gezi, c'est bien la défense des «modes de vie » (pour reprendre les catégories élaborées par les manifestants) qui est en jeu, et surtout la place de ces modes de vie dans l'espace public. Les manifestants ne se sont pas seulement élevés contre l'ordre moral religieux, mais également contre l'ordre économique consumériste, en occupant des centres commerciaux par exemple. Ils entendent faire valoir leurs droits au respect de la liberté individuelle et réclament une orientation différente de l'aménagement des espaces urbains. En cela, ces manifestations sont l'expression d'un droit à la ville, dans le sens de Lefebvre, car elles sont à la fois un cri - manifestation contre l'ordre moral urbain - et une exigence - construire la ville pour tous (Lefebvre, 1968 ; Harvey, 2011).

En Turquie, ce droit à la ville passe par une lutte contre la standardisation des espaces publics, qu'il s'agisse des parcs ou des malls, tous deux standardisés et répétés partout, présentés comme les symboles de la salubrité et de la réussite sociale. Pour les manifestants les plus conscientisés, la ville ne doit pas être un simple espace stratégique d'accumulation du capital (ce qui est le cas du mode de production actuel de la ville en Turquie) mais un espace de vie à habiter, et à habiter bien, en ayant un accès raisonnable à tout ce qui est nécessaire pour mener une vie urbaine décente. Selon Purcell, une des marques du droit à la ville est le droit d'appropriation, c'est-à-dire le droit à être physiquement présent dans un espace matériel déjà existant (Purcell, 2008). Les manifestants en Turquie ont cherché par tous les moyens à occuper l'espace public, à se l'approprier pour revendiquer leur droit à l'occuper. Occuper l'espace physique en premier lieu, en campant sur le place Taksim, en manifestant dans les rues, en discutant sur des marches ; mais également occuper l'espace visuel (drapeaux, lumières) et l'espace sonore (concert de casseroles), les trois principaux éléments constitutifs de l'espace public perçu. Boire un verre d'alcool dans la rue à la nuit tombée en chantant avec ses amis est ainsi devenu une marque de défiance au pouvoir et un acte fort de résistance par l'appropriation totale de l'espace public.

EchoGéo, Sur le Vif | 2013 


\section{BIBLIOGRAPHIE}

Artık Ö., 2013. La Turquie, retour au Moyen-Orient. Hérodote, $\mathrm{n}^{\circ}$ 148, p. 33-45.

Auyero J., 2005.L'espace des luttes. Topographie des mobilisations collectives. Actes de la recherche en sciences sociales, 2005/5, $\mathrm{n}^{\circ}$ 160, p. 122-132.

Batuman B., 2013. Minarets without mosques: limits to the urban politics of neo-liberal Islamism. Urban Studies, Vol. 50, Nº 6, p. 1097-1113.

Bonny Y., Ollitrault S., Keerle R., Le Caro Y., 2011. Espaces de vie, espaces enjeux. Entre investissements ordinaires et mobilisations politiques. Rennes, Presses Universitaires de Rennes, 407 p.

Brenner N., Theodore N., 2002. Cities and the geography of actually existing neoliberalism. Antipode, Vol. 34, N ${ }^{\circ}$, p. 349-379.

Candelier-Cabon M., Montabone B., 2009.Istanbul, une internationalisation forcée ? EchoGéo [En ligne], Sur le Vif, mis en ligne le 02 décembre 2009, URL : http://echogeo.revues.org/11523

Cankat A., 2010. Gecekondu : un projet urbain en acte et en puissance. Urbanisme, n 374, p. 60-61.

Dikeç M., 2013. Fraudulent democracy and urban stasis in Turkey. Society and Space, http:// societyandspace.com/2013/06/14/commentary-by-mustafa-dikec-fraudulent-democracy-andurban-stasis-in-turkey/

Dilek Y., 2012. La nouvelle bourgeoisie islamique. Le modèle turc. Paris, Presses Universitaires de France.

Erkip F., 2003. The shopping mall as an emergent public space in Turkey. Environment and Planning A, Vol. 35, p. 1073-1093.

Erkip F., 2005. The rise of the shopping mall in Turkey: the use and appeal of a mall in Ankara. Cities, Vol. 22, $\mathrm{N}^{\circ}$ 2, p. 89-108.

Gould R., 1995. Insurgent Identities: Class, Community and Protest in Paris from 1848 to the Comune. The University of Chicago Press, $260 \mathrm{p}$.

Harvey D., 2008 (trad. Fr). Géographie de la domination. Editions Les Prairies ordinaires, Paris.

Harvey D., 2011 (trad. Fr). Le capitalisme contre le droit à la ville. Néolibéralisme, urbanisation, résistances. Paris, Editions Amsterdam, 93 p.

Karaman O., 2008. Urban pulse - (Re)making space for globalization in Istanbul. Urban Geography, Vol. 29, $\mathrm{N}^{\circ} 6$, p. 518-525.

Kuyucu T., Ünsal Ö, 2010. Urban transformation as State-led property transfer: an analysis of two cases of urban renewal in Istanbul. Urban Studies, Vol. 47, N 7, p. 1479-1499.

Lefebvre H., 1968. Le droit à la ville. Paris, Anthropos (plusieurs rééditions).

Lefebvre H., 1970. La révolution urbaine, Paris, Gallimard, coll. Idées.

Mathieu L., 2011. Territoires et mises en espace des mobilisations. In Bonny Y., Ollitrault S., Keerle R., Le Caro Y., 2011, Espaces de vie, espaces enjeux. Entre investissements ordinaires et mobilisations politiques, Rennes, Presses Universitaires de Rennes, p. 185-195. 
Morange M., 2011. Droit à la ville, néolibéralisme et Etat développemental au Cap. Justice Spatiale | Spatial Justice, $\mathrm{n}^{\circ} 4$.

Öktem K., Kadığlu A., Karlı M., 2012. Another Empire? A Decade of Turkey's Foreign Policy under the Justice and Development Party. Istanbul, Bilgi University Press.

Öniș Z., 2012. The triumph of conservative globalism: the political economy of the AKP era. Turkish Studies, Vol. 13, $\mathrm{N}^{\circ} 2$.

Pérouse J.F., 1999. Le nouvel ordre urbain du Refah: urbanisation, gestion urbaine et urbanisme à Istanbul depuis mars 1994. Les Annales de l'Autre Islam, nº 6 (Islam en Turquie), Inalco-Erism, p. 277-289.

Pérouse J.F., 2004. Les tribulations du terme gecekondu (1947-2004) : une lente perte de substance. Pour une clarification terminologique. European Journal of Turkish Studies [Online], 1 | 2004, URL : http://ejts.revues.org/117

Pérouse J.F., 2007. Istanbul entre Paris et Dubaï : mise en conformité internationale, nettoyages et résistances. In Berry-Chikhaoui I., Deboulet A., Roulleau-Berger L. (dir.), Villes Internationales. Entre tensions et réactions des habitants, Editions La Découverte, collection Recherches, Paris.

Pérouse J.F., 2013. Le parc Gezi : dessous d'une transformation très politique. Métropolitiques, 24 juin 2013. URL : http://www.metropolitiques.eu/Le-parc-Gezi-dessous-d-une.html

Petit C., 2011. Engagement militant et politisation des mobilisations au sein des oppositions urbaines à Istanbul. EchoGéo [En ligne], 16 | 2011, mis en ligne le 04 juillet 2011, URL : http:// echogeo.revues.org/12445

Purcell M., 2008. Recapturing Democracy: Neoliberalization and the Struggle for Alternative Urban Futures. New York, Routledge, 224 p.

Uzun B., Çete M., H. Palancıoğlu H.M., 2010. Legalizing and upgrading illegal settlements in Turkey. Habitat International, Vol. 34, N² 2, p. 204-209.

Uysal A., 2008. Un espace enjeu à Istanbul. Place de Taksim et les guerres du $1^{\circ}$ mai. Communication au colloque Espaces de vie, espaces enjeu : entre investissements ordinaires et mobilisations politiques, Rennes, Université Rennes 2 et IEP, 5-7 novembre 2008.

Yalman G.L., 2009. Transition to Neoliberalism. The Case of Turkey in the 1980s. Istanbul, Istanbul Bilgi University Press, $432 \mathrm{p}$.

Zhao D., 1998. Ecologies of social movements: student mobilization during the 1989 prodemocracy movement in Beijing. American Journal of Sociology, Vol. 103, № 6, p. 1493-1529.

Zukin S., 2010. Naked City: the Death and Life of Authentic Urban Places. Oxford University Press, New York, 294 p.

\section{NOTES}

1. Cet article a été élaboré dans le cadre du programme ANR SYSREMO (Géographie de la mondialisation : émergence d'un système régional au Moyen-Orient).

2. Cette estimation se base sur des relevés de presse et un parcours quotidien des pages facebook dédiées au mouvement «occupygezi ». À notre connaissance, aucun décompte de manifestants n'a été proposé, ce qui rend très aléatoire une éventuelle cartographie générale du mouvement. 3. De nombreuses vidéos, photographies, performances sont disponibles sur les médias alternatifs créés pendant le mouvement, dont : http://www.capul.tv/ 
4. On peut se référer à la page Wikipedia consacrée au mouvement, qui présente les événements de manière chronologique et exhaustive. https://en.wikipedia.org/ wiki/2013_protests_in_Turkey. Certains blogs sont également bien alimentés: http:// www.thepolisblog.org/2013_06_01_archive.html

5. On peut consulter le site de la « plateforme Taksim», http://www.taksimplatformu.com/

6. Le «Plan Prost pour Istanbul » est le premier plan d'aménagement d'ensemble proposé pour Istanbul en 1936 par l'urbaniste français Henri Prost, invité à travailler en Turquie par Mustafa Kemal Atatürk. Il propose une sauvegarde et une mise en valeur des principaux monuments de la ville, une limite en hauteur des constructions pour préserver le paysage urbain et la percée de grandes artères pour favoriser la circulation automobile.

7. Cité dans le Hürriyet Daily News, « Taksim is a site for ideological predominance », 3 juin 2013.

8. Témoignage reçu par le site http://penserclasser.wordpress.com/le dimanche 16 juin à 16h, de la part de S., une « amie francophone résidant à Beyoğlu ».

9. Ainsi lors de la campagne des législatives de 2011, les affiches de l'AKP proclamaient le nombre d'aéroports construits dans le pays, le nombre de kilomètres d'autoroutes dans telle province, le nombre de logements neufs dans telle ville, etc.

10. MIPIM : Marché International des Professionnels de l'Immobilier.

11. Voir l'analyse (partisane) qui en est faite sur le blog Tarlabaşıistanbul, http:// www.tarlabasiistanbul.com/2011/05/istiklal-demiroren/

12. Discours prononcé le 26 avril 2013, où Recep Tayip Erdogan vante les bienfaits de l'ayran (sorte de yaourt liquide) comme boisson nationale, qui selon lui a été remplacé de force par la bière.

13. Voir la video sur CNNTürk: http://video.cnnturk.com/2011/haber/3/10/ak-partiliyoneticiden-skandal-sozlerir

14. «L'ère des tulipes » de l'Empire ottoman va communément de 1717 à 1730 . Elle est considérée comme une période faste de l'Empire, du fait notamment d'une grande stabilité politique et militaire et de l'ouverture culturelle sur l'Europe baroque.

\section{RÉSUMÉS}

L'article cherche à comprendre les raisons des événements du printemps 2013 en Turquie. Il montre que les manifestations ne sont pas uniquement dues à la volonté de défendre un espace vert menacé mais qu'elles sont la réponse de la population laïque turque à l'évolution des modes de faire la ville et au contrôle de l'espace public par le parti au pouvoir. Via un néolibéralisme économique, un conservatisme religieux et un néo-ottomanisme culturel, l'AKP (Parti de la Justice et du Développement) cherche en effet à imposer un ordre moral urbain que récusent les manifestants. Les émeutes de juin 2013 revendiquent ainsi un droit à la ville, qui s'exprime avant tout par la liberté d'appropriation des espaces publics.

This article seeks to understand the reasons of the violent demonstrations in Turkish cities in June 2013. It demonstrates that the defense of a public green space in the heart of Istanbul is not the main driver of the revolt, but moreover the latter is the response of the secular people to the evolution of the ways of producing the city and of controlling the public spaces by the islamist ruling party AKP. Contesting at the same time neoliberalism, conservatism and neo-ottomanism, 
the demonstrators strike against the new urban moral order of the AKP, claiming for freedom in public spaces and far beyond for an effective right to the city.

\section{INDEX}

Mots-clés : droit à la ville, contestation urbaine, espace public, conflit, Turquie

Keywords : right to the city, urban protests, public space, conflict, Turkey

\section{AUTEUR}

\section{BENOIT MONTABONE}

Benoit Montabone (benoit.montabone@univ-rennes2.fr) est Maître de conférences en Géographie à l'université Rennes 2. Il est membre du laboratoire ESO-Rennes (UMR 6590 ESO). Il a récemment publié :

- Montabone B., 2013. Périphéries exploitées ou relais indispensables ? Les petites villes du département d'Izmir (Turquie) dans le projet métropolitain. Annales de Géographie, 1/2013, n - 689, p. 24-46. http://www.cairn.info/revue-annales-de-geographie-2013-1-page-24.htm - Montabone B., 2012. La région, entre attentes européennes et trajectoire nationale : les « agences de développement » en Turquie. Territoire en mouvement, $n^{\circ} 16$, p. 71-85. http:// tem.revues.org/1899. 